\title{
Attention Networks in the Parietooccipital Cortex Modulate Activity of the Human Vestibular Cortex during Attentive Visual Processing
}

\author{
DSebastian M. Frank, ${ }^{1,2}$ Maja Pawellek, ${ }^{1}$ Lisa Forster, ${ }^{1}$ Berthold Langguth, ${ }^{3}$ Martin Schecklmann, ${ }^{3}$ \\ and $\odot$ Mark W. Greenlee ${ }^{1}$ \\ ${ }^{1}$ Institute for Experimental Psychology, University of Regensburg, Regensburg 93053, Germany, ${ }^{2}$ Department of Cognitive, Linguistic, and Psychological \\ Sciences, Brown University, Providence, Rhode Island 02912, and ${ }^{3}$ Department of Psychiatry and Psychotherapy, University of Regensburg, Regensburg \\ 93053, Germany
}

Previous studies in human subjects reported that the parieto-insular vestibular cortex (PIVC), a core area of the vestibular cortex, is inhibited when visual processing is prioritized. However, it has remained unclear which networks in the brain modulate this inhibition of PIVC. Based on previous results showing that the inhibition of PIVC is strongly influenced by visual attention, we here examined whether attention networks in the parietooccipital cortex modulate the inhibition of PIVC. Using diffusion-weighted and resting-state fMRI in a group of female and male subjects, we found structural and functional connections between PIVC and the posterior parietal cortex (PPC), a major brain region of the cortical attention network. We then temporarily inhibited PPC by repetitive transcranial magnetic stimulation (rTMS) and hypothesized that the modulatory influence of PPC over PIVC would be reduced; and, as a result, PIVC would be less inhibited. Subjects performed a visual attentional tracking task immediately after rTMS, and the inhibition of PIVC during attentive tracking was measured with fMRI. The results showed that the inhibition of PIVC during attentive tracking was less pronounced compared with sham rTMS. We also examined the effects of inhibitory rTMS over the occipital cortex and found that the visual-vestibular posterior insular cortex area was less activated during attentive tracking compared with sham rTMS or rTMS over PPC. Together, these results suggest that attention networks in the parietooccipital cortex modulate activity in core areas of the vestibular cortex during attentive visual processing.

Key words: attention; cross-modal inhibition; multisensory conflicts; parietooccipital cortex; rTMS; visual-vestibular interaction

\section{Significance Statement}

Although multisensory integration is generally considered beneficial, it can become detrimental when cues from different senses are in conflict. The occurrence of such multisensory conflicts can be minimized by inhibiting core cortical areas of the subordinate sensory system (e.g., vestibular), thus reducing potential conflict with ongoing processing of the prevailing sensory (e.g., visual) cues. However, it has remained unclear which networks in the brain modulate the magnitude of inhibition of the subordinate sensory system. Here, by investigating the inhibition of the vestibular sensory system when visual processing is prioritized, we show that attention networks in the parietooccipital cortex modulate the magnitude of inhibition of the vestibular cortex.

\section{Introduction}

The integration of signals from different sensory systems is generally considered beneficial, as it makes perception more precise

\footnotetext{
Received Aug. 11, 2019; revised Nov. 6, 2019; accepted Nov. 7, 2019.

Author contributions: S.M.F., M.P., L.F., B.L., M.S., and M.W.G. designed research; S.M.F., M.P., and L.F. performed research; S.M.F. analyzed data; S.M.F. wrote the first draft of the paper; S.M.F., M.P., L.F., B.L., M.S., and M.W.G. edited the paper; S.M.F., M.P., L.F., B.L., M.S., and M.W.G. wrote the paper.

The work was supported by Deutsche Forschungsgemeinschaft Grants INST 89/393-1 and GR 988/25-1. We thank Nexstim PIc. for providing the electric-field guided neuronavigation rTMS system; and Christian Renner (University of Regensburg workshop) for constructing and maintaining the caloric vestibular stimulation device.

The authors declare no competing financial interests.
}

and contributes to adaptive behavior. However, signals from different senses are often in conflict, which renders their integration difficult or impossible. Imagine being a passenger inside a moving car while focusing on a computer screen on your lap. Central vision would signal a stable visual environment, whereas the vestibular sense would signal accelerations or decelerations of the car, resulting in a visual-vestibular conflict.

Correspondence should be addressed to Sebastian M. Frank at sebastian_frank@brown.edu. https://doi.org/10.1523/JNEUROSCl.1952-19.2019

Copyright $\odot 2020$ the authors 
Previous results (Brandt et al., 1998; Deutschländer et al., 2002; Kleinschmidt et al., 2002) suggest that visual-vestibular conflicts are minimized by cross-modal inhibition of the subordinate sensory system. For instance, when visual processing is prioritized, the parieto-insular vestibular cortex (PIVC), a core area of the human and nonhuman primate vestibular cortex (Guldin and Grüsser, 1998; Lopez and Blanke, 2011; Frank and Greenlee, 2018), is inhibited, thus reducing the possibility of conflicting vestibular signals interrupting the cortical processing of the visual cue (Brandt et al., 1998; Brandt and Dieterich, 1999). Cross-modal adaptation aftereffects in the vestibular system after prolonged stimulation with visual optic flow are thought to arise from this inhibition of the vestibular system (Cuturi and MacNeilage, 2014).

In a previous study (Frank et al., 2016a), we observed that attention directed toward visual processing strongly influenced the inhibition of PIVC. We measured activity in PIVC with fMRI while subjects performed a visual attentional tracking task. In this task, participants were requested to follow a subset of moving visual targets among moving distractors with their attention. The results showed that the inhibition of PIVC increased dramatically in magnitude when subjects attentively tracked the visual targets compared with passive viewing of the same stimuli. Interestingly, opposite results were observed for posterior insular cortex (PIC), a visual-vestibular area located posterior to PIVC (Frank et al., 2014, 2016b; Billington and Smith, 2015; Roberts et al., 2017; Frank and Greenlee, 2018; Schindler and Bartels, 2018), such that activity in PIC increased during attentive tracking compared with passive viewing.

These results agree with psychophysical findings showing that attention directed either to the visual or vestibular sensory modality suppresses sensations related to the nonattended sensory modality (Berger and Bülthoff, 2009). Thus, attention might play a critical role in modulating activity of the vestibular cortex when visual processing is prioritized.

The aims of this study were to clarify whether visual attention modulates activity of the vestibular system and to identify the neuronal origin of this attentional modulation. We conducted a series of experiments using psychophysics, fMRI and structural MRI, and inhibitory repetitive transcranial magnetic stimulation (rTMS). In an initial behavioral experiment, we addressed the question of whether visual attention modulates the magnitude of vestibular suppression. In this experiment, subjects performed a visual attentional tracking task during caloric vestibular stimulation (CVS) and rated the magnitude of their vestibular sensations during attentive tracking compared with passive viewing of the same visual stimuli. In a subsequent experiment, we measured the cross-modal effects of visual attention on ongoing activity in PIVC and PIC with fMRI. In a further experiment, we examined the structural and functional connectivity of PIVC and PIC using diffusion tensor imaging (DTI) and fMRI resting-state measurements. As we observed connectivity between PIVC/PIC and posterior parietal cortex (PPC), a major brain region of the cortical attention network, we conducted a final experiment and examined whether inhibitory rTMS over PPC reduced the inhibition of PIVC and the activation of PIC during visual attentional tracking. We compared the results of inhibitory rTMS over PPC to conditions of inhibitory rTMS over occipital cortex (OC) and sham rTMS. Across experiments, our results suggest that the activity in core areas of the vestibular cortex can be strongly modulated by visual attention, originating from attention networks in the parietooccipital cortex.

\section{Materials and Methods}

Participants. A total of $n=23$ subjects ( 18 females) with a mean ( \pm SE) age of $24 \pm 1$ years participated in the study. All subjects were righthanded as determined by the Edinburgh Handedness Inventory (Oldfield, 1971). The mean $( \pm \mathrm{SE})$ right handedness score was $93.9 \pm 2.45$ $(\min =54, \max =100)$. A subset of $n=15$ subjects participated in the behavioral experiment. A subset of $n=20$ subjects (including 12 subjects from the behavioral experiment) participated in the MRI experiments without rTMS. Finally, a subset of $n=15$ subjects from the MRI experiments volunteered to participate in the rTMS experiments. The study was approved by the local ethics board at the University of Regensburg. Subjects gave informed written consent before participation. Subjects were familiarized with the TMS-procedure, and possible contraindications to TMS were checked following recommendations by the Safety of TMS Consensus Group (Rossi et al., 2009).

Experimental design. The study consisted of four experiments. First, we used psychophysics to examine whether vestibular sensations can be modulated by visual attention. Next, we investigated the neuronal effects of visual attention in core areas of the vestibular cortex using fMRI. Afterward, we addressed the question of whether core areas of the vestibular cortex are structurally and functionally connected with cortical attention networks. Finally, we tested whether these attention networks modulate the inhibition of the vestibular cortex by using fMRI and inhibitory rTMS. Separate fMRI localizer experiments were conducted to define ROIs in the vestibular and occipital cortex. The attentional tracking task that was used in the major experiments is described next, followed by a detailed overview of the procedure for each experiment.

Attentional tracking task. An attentional tracking task (Pylyshyn and Storm, 1988; Culham et al., 2001; Frank et al., 2016a) was used to measure activity in core areas of the vestibular cortex during attentive visual processing. In this task, subjects were presented with displays of randomly moving stimuli and attentively tracked a subset of those stimuli. The effects of attention were measured by comparing the attentive tracking condition with a passive viewing condition of the same motion displays. The motion displays consisted of a set of four white disks (diameter: $0.6^{\circ}$ ) moving randomly with a speed of $3.5^{\circ} / \mathrm{s}$ in the lower left visual quadrant (diameter of quadrant $=8.5^{\circ}$ ). The lower left visual quadrant was chosen because of its representation in the upper right OC, which could be targeted by TMS. Each tracking trial started with a 2-slong cueing phase during which all disks remained stationary. During this phase, two of four disks were highlighted in green to denote them as targets. The other two disks remained white and served as distractors. After the cueing phase, the targets turned white and were therefore physically indistinguishable from the distractors. Then, all disks began to move within the lower left visual quadrant. Disks never collided or overlapped and were repelled from the invisible borders of the lower left visual quadrant and from central fixation. Subjects tracked the targets with their attention while maintaining fixation on a cross presented within a gray disk (diameter $=0.8^{\circ}$ ) at the center of the screen. If subjects lost track of a target, they were instructed to track another disk to keep the number of tracking targets stable across each tracking trial. After $14 \mathrm{~s}$, all disks stopped moving, and one of the disks was highlighted in green. Subjects were given $2 \mathrm{~s}$ to indicate whether this highlighted disk was a target or a distractor. After the response phase, subjects received feedback about the correctness of their response. To this aim, the central fixation cross either turned to green for a correct response or to red for an incorrect response for $2 \mathrm{~s}$. Each trial was $20 \mathrm{~s}$ long. The passive viewing condition was identical with the attentive tracking condition, except that no disks were denoted as targets in the cueing phase or highlighted during the response phase (i.e., all disks were presented in white during the entire trial and no response was required). Furthermore, central fixation did not change color for response feedback.

Behavioral experiment. In this experiment, we examined whether attention modulates the suppression of vestibular sensations when visual processing is prioritized. Specifically, visual motion cues in form of the previously described attentional tracking task were combined with CVS (see Fig. 1a). Participants either passively viewed the visual motion displays or attentively tracked a subset of the moving stimuli with their 
attention. Subjects were in supine position on a mat on the floor in a psychophysics laboratory. Their heads were slightly elevated with a pillow by $20^{\circ}-30^{\circ}$ to move the horizontal ear canals approximately into vertical position for optimal CVS (Barnes, 1995). A computer screen (screen size $=35^{\circ} \times 26^{\circ}$ ) was mounted above subjects' heads (viewing distance $=60 \mathrm{~cm}$ ) on the bottom of a table. Dark curtains were attached to each side of the table to minimize any visual input. The room was darkened, and the only visual stimulation occurred on the computer screen. Two CVS conditions were used: (1) hot left and cold right and (2) cold left and hot right (hot $=45^{\circ} \mathrm{C}$, cold $=22^{\circ} \mathrm{C}$ ). CVS was conducted with a custom-built device (Frank and Greenlee, 2014), which is described in detail below (see Localizer for vestibular area PIVC). The caloric conditions were combined with three different visual conditions: (1) eyes closed (vestibular-only condition), (2) eyes open and passive viewing of visual motion (passive viewing condition), and (3) eyes open and attentional tracking of visual motion (attentive tracking condition) (see Fig. 1a). The combination of caloric-vestibular and visual conditions was counterbalanced for each subject. During passive viewing and attentive tracking, three successive visual stimulation trials were presented on each CVS trial block. In the attentive tracking condition, subjects reported at the end of each tracking trial whether the highlighted disk in the response phase was a target or a distractor. Participants responded by pressing one of two buttons for target or distractor on a button box. Additionally, subjects monitored their vestibular sensations. Each CVS trial block was $80 \mathrm{~s}$ long. The first and last $10 \mathrm{~s}$ served as on- and offramps, during which temperatures were switched from binaural warm baseline $\left(36.5^{\circ} \mathrm{C}\right)$ to caloric stimulation (on-ramp) or vice versa (offramp) (Frank and Greenlee, 2014). During on- and off-ramps, subjects either maintained fixation on the central fixation spot in the passive viewing and attentive tracking conditions or kept their eyes closed in the vestibular-only condition. After each caloric-vestibular trial, participants verbally reported on their vestibular sensations during the stimulation to the experimenter who entered the responses into the computer. Subjects were asked to base their judgments about vestibular sensations on the central $60 \mathrm{~s}$ of the CVS (i.e., excluding on- and off-ramps with temperature changes). Specifically, they reported the overall strength of their self-motion sensations on a Likert scale ranging from 0 (no self-motion) to 10 (strong self-motion with feelings of vertigo and discomfort) in integer steps. Values $>5$ indicated self-motion with increasing sensations of discomfort and vertigo. Furthermore, subjects indicated the direction of rotation (pitch, roll, yaw, or a combination). A total of 24 CVS trial blocks were conducted ( 8 for each visual condition), resulting in an experiment duration of $\sim 45 \mathrm{~min}$. Trial blocks were presented in random order.

We hypothesized that vestibular sensations would be most pronounced when subjects kept their eyes closed (Deutschländer et al., 2002; Dieterich et al., 2003) and reduced when subjects passively processed the visual stimuli, indicative of vestibular suppression during visual processing (Brandt et al., 1998). Furthermore, we predicted that these suppression effects on vestibular sensations would be larger when subjects processed the visual stimuli attentively in the attentional tracking condition, indicating that the magnitude of vestibular suppression can be modulated by attention directed toward visual processing.

fMRI experiment without $r$ TMS. An fMRI experiment was conducted to measure cross-modal activity changes in core areas of the vestibular cortex (PIVC and PIC, respectively) during visual attentional tracking and passive viewing. Two runs of the previously described attentional tracking task in the lower left visual quadrant were conducted (run length $=16 \mathrm{~min}$ ). Each run consisted of 12 trials for attentive tracking and 12 trials for passive viewing that were presented in random order. Each trial was $20 \mathrm{~s}$ long and was followed by a 20-s-long blank baseline during which participants kept their eyes open to avoid that eye closure during baseline would activate the vestibular cortex (see Marx et al., 2004). No caloric stimuli were applied to avoid additional modulations of brain activity by vestibular input (Deutschländer et al., 2002; Frank et al., 2014). Based on the results of our previous study using a similar task (Frank et al., 2016a), we predicted that attentive visual processing would increase the magnitude of inhibition of PIVC, as indicated by a negative
BOLD response, while simultaneously increasing the magnitude of activation of PIC (as indicated by a positive BOLD response).

Structural and functional connectivity experiment. To examine whether PIVC and PIC are structurally and functionally connected with cortical attention networks, we conducted MRI measurements for structural and functional connectivity. Structural connectivity was measured by means of DTI (see below). Functional connectivity was measured with a restingstate fMRI scan during which participants maintained central fixation. One run was conducted (run duration $=10 \mathrm{~min}$ ). The structural and functional connectivity of PIVC was computed by using PIVC as the seed region. A separate analysis was conducted with PIC as the seed region.

fMRI experiment with $r T M S$. In this experiment, inhibitory rTMS over different brain regions was conducted before the attentional tracking task that subjects then performed during fMRI measurements. The goal was to discover brain networks modulating cross-modal activity changes in the vestibular cortex when visual processing is prioritized. The rationale was that inhibitory, low-frequency $(1 \mathrm{~Hz})$ rTMS over brain regions that modulate activity in the vestibular cortex would temporarily reduce their modulatory influence after rTMS (Kobayashi and Pascual-Leone, 2003; Ruff et al., 2009; Thut and Pascual-Leone, 2010; Rafique et al., 2015; Solomon-Harris et al., 2016). In different sessions, we targeted two different sites with inhibitory rTMS: the PPC and the OC, which were both defined in separate fMRI localizer experiments (see Fig. 4). A control region located halfway between PPC and OC was chosen for sham rTMS. This sham control region was located in the higher-order visual cortex (Brodmann area 18), at the border between the occipital and the parietal cortex (see Fig. 4a). Inhibitory rTMS was administered over the three sites in separate sessions, which were at least 1 week apart to avoid any possible carryover effects of rTMS from one session to another. The assignment of stimulation sites to different sessions was counterbalanced across subjects. The definition of rTMS target regions by means of structural MRI and fMRI and the exact procedure of inhibitory rTMS are described in the following sections.

Definition of rTMS target region in the posterior parietal cortex. A previous study using DTI in human subjects (Wirth et al., 2018) reported structural connections between PIVC and PPC, which could find support for a role of the parietal cortex in modulating the inhibition of PIVC during attentive visual processing (Frank and Greenlee, 2018). Therefore, we used PPC as target site for inhibitory rTMS. To determine the specific site in the parietal cortex that is connected with PIVC in individual subjects, we conducted MRI measurements for structural and functional connectivity (see above). The part of the parietal cortex that was structurally and functionally connected with PIVC in individual subjects was used as the target site for inhibitory rTMS (for a group analysis, see Fig. $3 a$ ). On average, across subjects, a subsection of the intraparietal sulcus and the surrounding cortex was structurally and functionally connected with PIVC (corresponding to Brodmann areas 7 and 19; see Fig. $4 b, c$ ).

We also computed the structural and functional connectivity of PIC and observed connectivity between PIC and PPC (see Fig. $3 b$ ). The connectivity of PIC overlapped in the intraparietal sulcus with the connectivity of PIVC (see Fig. 3a). Since the main focus of this study was PIVC, we used the connectivity results from PIVC for the definition of the rTMS target site in PPC.

Definition of rTMS target region in the occipital cortex. The target region for rTMS in the OC consisted of the representation of the lower left visual quadrant, where the attentional tracking task was performed (see Fig. $4 e-g$ ). To localize this representation, fMRI measurements of 12 -s-long blocks of stimulation with flickering checkerboards (flicker frequency = $8 \mathrm{~Hz}$ ) in the lower left visual quadrant were contrasted with 12-s-long blocks of stimulation in the other three quadrants. Each block with stimulation was followed by a 12-s-long blank baseline period. Stimulation conditions were presented in random order, and there were five blocks for each stimulation condition. Subjects maintained central fixation and pressed a button on the button box when they detected a color change of the fixation cross, which occurred occasionally. One run was conducted (run duration $\sim 4 \mathrm{~min}$ ).

We confined the representation of the lower left quadrant to the early visual cortex, also referred to as V1 (Brodmann area 17). To define V1, 
phase-encoded retinotopic mapping was conducted. To this aim, a bowtie-shaped flickering checkerboard rotated in clockwise or counterclockwise directions across the screen. During each rotation cycle, 18 locations were stimulated for $3 \mathrm{~s}$ each. In total, there were 12 cycles for clockwise and counterclockwise directions, respectively, which were conducted in separate fMRI runs. Each fMRI run was $\sim 11 \mathrm{~min}$ long. The alternating representations of the vertical and horizontal meridians demarcate the borders between the visual areas V1, V2, and V3.

rTMS procedure. Target regions for rTMS were localized on each participant's skull by means of neuronavigation using the Nexstim system. The TMS targets as well as the optimal orientation of the TMS coil were marked on a cap for each participant individually. For rTMS, a Mag Pro Stimulator (MagVenture) with a butterfly-shaped magnetic coil was used. To determine the optimal intensity of TMS for individual subjects, the motor threshold was measured beforehand. To this aim, single TMS pulses were applied over the right motor cortex while measuring motor evoked potentials in the left index finger using electromyography. An initial TMS intensity of 50\% was used and reduced until 4 motor evoked potentials of 8 TMS pulses could still be detected (Schecklmann et al., 2012; Rossini et al., 2015). For rTMS, the TMS intensity was set to $110 \%$ of each participant's individual motor threshold.

The rTMS sessions with subsequent fMRI scanning were conducted as follows: participants were seated in a chair, and the TMS coil was placed over the target region using position and orientation parameters determined by neuronavigation (see above). For sham rTMS, the coil was positioned with the coil's reverse side on the skull surface, which reduces the stimulation intensity to one-sixth of the normal intensity (Van Doren et al., 2015; Engel et al., 2017). The clicking sounds and vibration of the coil accompanying real stimulation were indistinguishable in the sham rTMS condition. A total of 1800 biphasic TMS pulses with a frequency of $1 \mathrm{~Hz}$ were administered, resulting in a duration of rTMS of $30 \mathrm{~min}$. With this frequency, we expected to inhibit the target region for at least $20 \mathrm{~min}$ after stimulation end (Kobayashi and Pascual-Leone, 2003; Thut and Pascual-Leone, 2010). Participants were instructed to close their eyes during stimulation. An experimenter remained in the room with the subjects during rTMS and checked on their well-being every $5 \mathrm{~min}$. Subjects indicated to the experimenter by gesturing ("thumbs up") if everything was fine. When the last $30 \mathrm{~s}$ of rTMS began, subjects were alerted by the experimenter to get prepared for the fMRI measurements. Thereafter, the subjects were guided by the experimenter to the MRI scanner located in the room next door, were then positioned on the MRI table, and were moved into the scanner. The mean time between the end of rTMS and the beginning of the fMRI measurements across subjects was $1: 54 \mathrm{~min}(\mathrm{SE}=0.11 \mathrm{~min})$ for sham $\mathrm{rTMS}, 1: 58 \mathrm{~min}(\mathrm{SE}=0.09 \mathrm{~min})$ for parietal $\mathrm{rTMS}$, and 1:53 $\mathrm{min}(\mathrm{SE}=0.09 \mathrm{~min})$ for occipital $\mathrm{rTMS}$. There were no significant differences in elapsed time between the three rTMS conditions (Friedman's ANOVA, $\chi_{(2)}^{2}=4.31, p=0.12$ ). One fMRI run, including 12 passive viewing and 12 attentive tracking trials presented in random order, was conducted (same task as in the preceding fMRI session without prior rTMS; see above). The run duration was $16 \mathrm{~min}$.

After scanning, subjects were asked to rate their experiences during rTMS on an integer scale from 0 (very unpleasant) to 10 (very pleasant). Subjects gave the following mean ratings for sham rTMS: $6.4(\mathrm{SE}=0.4)$; for parietal rTMS: $5.3(\mathrm{SE}=0.5)$; and for occipital rTMS: $5.2(\mathrm{SE}=0.5)$. The ratings were significantly different between the rTMS conditions (Friedman's ANOVA, $\chi_{(2)}^{2}=10.2, p=0.006$ ). Post hoc Wilcoxon signedrank tests showed that the sham session was rated to be more pleasant than rTMS over PPC $(Z=-2.03, p=0.04, r=-0.37)$ and rTMS over OC $(Z=-2.50, p=0.01, r=-0.46)$. rTMS over PPC and OC did not evoke significantly different pleasantness ratings $(Z=-0.51, p=0.61$, $r=-0.09)$. Furthermore, participants were asked whether they experienced any side effects during rTMS. No side effects of rTMS in any session were evident, except for slight scalp pain sensations or a mild headache in one session reported by 2 subjects.

Localizer for vestibular area PIVC. Area PIVC was localized in each participant beforehand by means of CVS during fMRI following previous descriptions (see Frank et al., 2016b). Vestibular stimulation was conducted with a custom-made MRI-safe binaural CVS device. The device consisted of several components, which are described in detail previously
(Frank and Greenlee, 2014). In brief, hot and cold water was stored in two barrels located outside the scanner room, and tempered water was pumped via tubes to the left and right ear canals of the participant in the MRI scanner. Small glass-made pods installed in the MRI-headphone system transmitted the temperatures of the water to the ear canals while the water remained inside the glass pods. The water was returned via separate tubes to a collecting barrel in the scanner control room. Three different temperature states were provided to the ear canals: (1) hot in the left and cold in the right, (2) cold in the left and hot in the right, and (3) warm in both. Hot and cold were used for CVS. Warm was used for baseline. The same temperatures as in the behavioral experiment were used.

CVS trials with hot in one ear and cold in the other ear were presented in random order. Each CVS trial lasted $60 \mathrm{~s}$ and was followed by a 60 -slong baseline with warm in both ears. A total of 20 trials were conducted in a single fMRI-run (five with hot left and cold right, five with cold left and hot right, and 10 warm baseline trials), resulting in a run-length of 20 min. Subjects kept their eyes closed and did not perform any task. After scanning, subjects reported on their vestibular sensations using a structured questionnaire (see Frank et al., 2016b). All subjects $(n=20)$ reported that they sensed self-motion during CVS. $N=19$ subjects described the sensation of self-motion as rotation that was, for a subset of $n=8$ subjects, accompanied by sensations of body sway. One participant indicated only sensations of body sway. $N=15$ participants described self-motion in the yaw direction that was mixed with roll or pitch directions for a subset of $n=6$ subjects. Four subjects experienced self-motion only in the roll direction, whereas 1 subject experienced a mixture of roll and pitch. For $n=11$ subjects, self-motion sensations were restricted to the head, whereas the remaining participants sensed self-motion of the whole body. None of the participants indicated any discomfort during CVS.

Temperature control experiment. The CVS device avoids somatosensory side effects because the water remains inside the closed-loop system. Furthermore, somatosensory stimulation from the presence of the pods inside the ear canal occurs in both the caloric-vestibular and baseline conditions. However, the different temperatures (hot, cold, warm) per se could induce additional activations that are unrelated to the vestibular effects. Specifically, this could be the case in the insula and in the parietal operculum (Craig et al., 2000), close to the location of PIVC (Lopez and Blanke, 2011; Frank and Greenlee, 2018). Therefore, we conducted a temperature-control experiment for which the stimulation pods were attached to the pinna during the fMRI measurements. With this setup, subjects could still clearly sense the differences in temperature, but no vestibular stimulation occurred. All other parameters were identical with the PIVC localizer experiment. None of the subjects $(n=20)$ reported any sensations of self-motion after the experiment.

Localizer for visual-vestibular area PIC. Area PIC was localized as described previously (see Frank et al., 2014, 2016b). During fMRI measurements, displays of dots moving in different translational directions $(1 \mathrm{~s}$ each direction, motion speed $=14 \%$ s) alternated with displays of static dots. Conditions of visual motion and static dots were presented in 12s-long blocks and 48 blocks in total ( 24 with visual motion, 24 with static dots) were used, resulting in a run length of $\sim 10 \mathrm{~min}$. The visual motion stimulus consisted of 200 white dots (diameter $=0.1^{\circ}$ ) that were placed at random locations across the dark screen. Each dot had a limited random lifetime between 167 and $333 \mathrm{~ms}$ and thereafter was replaced at a new location. Participants maintained central fixation and performed a color detection task on the fixation spot. One fMRI run was conducted.

Stimulus presentation. Stimuli were presented with the Psychophysics Toolbox (Brainard, 1997; Pelli, 1997) running in MATLAB (The MathWorks). Stimuli in the scanner were back-projected onto a translucent screen at the back of the scanner bore (screen size $=24^{\circ} \times 18^{\circ}$, viewing distance $=97 \mathrm{~cm}$ ). Subjects could see the screen with a mirror mounted on the MRI head coil.

Scanning parameters. The MRI data were collected on a Prisma 3 Tesla MRI scanner (Siemens). For the PIVC localizer, the temperature control experiment, and the PIC localizer, a 20-channel head coil was used and $\mathrm{T} 2{ }^{\star}$-weighted EPI data were collected with the following parameters: $\mathrm{TR}=2 \mathrm{~s}, \mathrm{TE}=30 \mathrm{~ms}$, flip angle $(\mathrm{FA})=90^{\circ}$, in-plane acquisition matrix 
$(\mathrm{AM})=64 \times 64,32$ axial slices, voxel size $=$ $3 \times 3 \times 3 \mathrm{~mm}$, interslice gap $=0.5 \mathrm{~mm}$. All other fMRI data were acquired with a 64channel head/neck coil and the following EPI parameters: $\mathrm{TR}=1 \mathrm{~s}, \mathrm{TE}=33 \mathrm{~ms}$, multiband factor $4, \mathrm{FA}=59^{\circ}, \mathrm{AM}=96 \times 96,48$ axial slices, voxel size $=2.5 \times 2.5 \times 2.5 \mathrm{~mm}$, no interslice gap. Anatomical scans were also collected with the 64-channel coil. For the highresolution T1-weighted anatomical scan of each participant's brain, we used an MPRAGE $\left(\mathrm{TR}=2.3 \mathrm{~s}, \mathrm{TE}=2.32 \mathrm{~ms}, \mathrm{FA}=8^{\circ}, \mathrm{AM}=\right.$ $256 \times 256,192$ sagittal slices, voxel size $=0.9 \times$ $0.9 \times 0.9 \mathrm{~mm}$, interslice gap $=0.45 \mathrm{~mm}$ ). Diffusion-weighted structural imaging data were acquired with a single-shot, spin-echo sequence with echo-planar readout $(\mathrm{TR}=3.9 \mathrm{~s}$, $\mathrm{TE}=78 \mathrm{~ms}$, multiband factor $2, \mathrm{FA}=90^{\circ}$, $\mathrm{AM}=106 \times 106,72$ axial slices, voxel size $=$ $2 \times 2 \times 2 \mathrm{~mm}$, no interslice gap). Diffusion was probed along 64 equally distributed orientations at b values $1000 \mathrm{~s} / \mathrm{mm}^{2}$ and 2000 $\mathrm{s} / \mathrm{mm}^{2}$. Nine volumes without diffusion weighting (b-zero) were interspersed.

MRI analysis. Each participant's high-resolution anatomical scan of the brain was reconstructed and inflated using Freesurfer (Martinos Center for Biomedical Imaging) (Dale et al., 1999; Fischl et al., 1999). fMRI data were preprocessed and analyzed with the FSFAST toolbox. Preprocessing included motion correction, coregistration to the reconstructed anatomical brain scan, smoothing with a 3D Gaussian kernel (FWHM $=3 \mathrm{~mm}$ for all scans with $2.5 \mathrm{~mm}^{3}$ voxel size; for all other scans, the smoothing kernel was set to $5 \mathrm{~mm}$ ), and intensity normalization. The coregistrations were carefully checked and manually corrected if necessary.

The fMRI data were analyzed with a GLM approach. For each analysis except the resting-state scan, a block-design was used. Each GLM contained motion correction parameters and a linear scanner drift predictor as regressors of no interest. The BOLD response was modeled using the SPM canonical hemodynamic response function.

The GLM for the PIVC localizer contained three regressors of interest for the two CVS conditions and the baseline condition. The first $10 \mathrm{~s}$ of each trial served as ramp for temperature changes and was not included in the analysis. Thus, each regressor of interest modeled a period of $50 \mathrm{~s}$ for each trial and condition. PIVC was defined by contrasting CVS (both caloric stimulation conditions combined) with baseline.

There were two regressors of interest for the visual motion and static baseline blocks in the GLM of the PIC localizer scan. PIC was defined by contrasting visual motion with baseline.

The localizer experiment for the representation of the lower left visual quadrant in the OC was analyzed by constructing two regressors of interest for stimulation in the lower left quadrant and stimulation in the other quadrants. The representation of the lower left quadrant was defined by contrasting stimulation in this quadrant with stimulation in all other quadrants. Furthermore, the representation of the lower left quadrant was confined to the early visual cortex, which was determined by phase-encoded retinotopic mapping.

For the attentional tracking task, three regressors of interest were constructed: one regressor for the passive viewing condition, another one for the attentional tracking condition, and a third one for the baseline condition. The regressors for passive viewing and attentional tracking included the 14-s-long period during which disks moved while subjects either passively viewed or attentively tracked them. Three additional regressors of no interest were included for cueing, response, and feedback phases ( $2 \mathrm{~s}$ each). For the primary analysis, only the tracking trials where subjects responded correctly were used. A regressor of no interest covered the tracking trials with incorrect responses (if any). For the attentional tracking experiment following rTMS, we conducted an additional control analysis, for which all tracking trials were included in the analysis
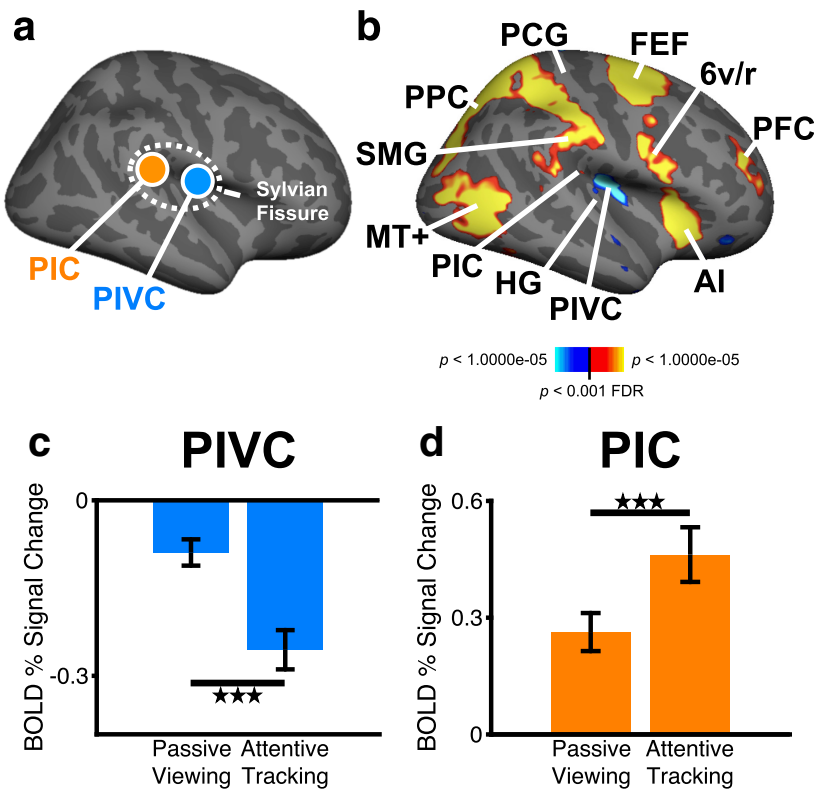

Figure 2. Cross-modal effects of visual attention on the BOLD response in core areas of the vestibular cortex. $\boldsymbol{a}$, Location of the PIVC and the PIC in the mid-posterior Sylvian fissure (displayed on the inflated right hemisphere of a template brain). $\boldsymbol{b}$, Whole-brain random-effects group analysis ( $n=20$ subjects) for the contrast attentive tracking versus passive viewing. Red-yellow represents stronger activity during attentive tracking compared with passive viewing. Blue-white represents the reverse contrast. $6 \mathrm{v} / \mathrm{r}$, Ventral/rostral area 6 (derived from the anatomical segmentation by Glasser et al., 2016); Al, anterior insula; FEF, frontal eye fields; HG, Heschl's gyrus (corresponding to the primary auditory cortex); MT + , human area MT+ (V5); $P C G$, postcentral gyrus (corresponding to the primary somatosensory cortex); PFC, prefrontal cortex (dorsolateral part); SMG, supramarginal gyrus. $\boldsymbol{c}, \boldsymbol{d}$, Mean ( \pm SE) activity measured as BOLD percentage signal change in PIVC and PIC for the same subjects as in $\boldsymbol{b}$ during passive viewing and attentive tracking of visual motion cues. PIVC and PIC were defined in independent localizer experiments (for details, see Materials and Methods). Zero on the $y$ axis indicates activity during blank baseline while participants kept their eyes open. Significant differences between passive viewing and attentive tracking conditions: ${ }^{* * *} p<0.001$.

regardless of correct or incorrect subject response. This was done to rule out the possibility that differences in the number of analyzed trials had contributed to any differences between rTMS conditions found in the primary analysis. 


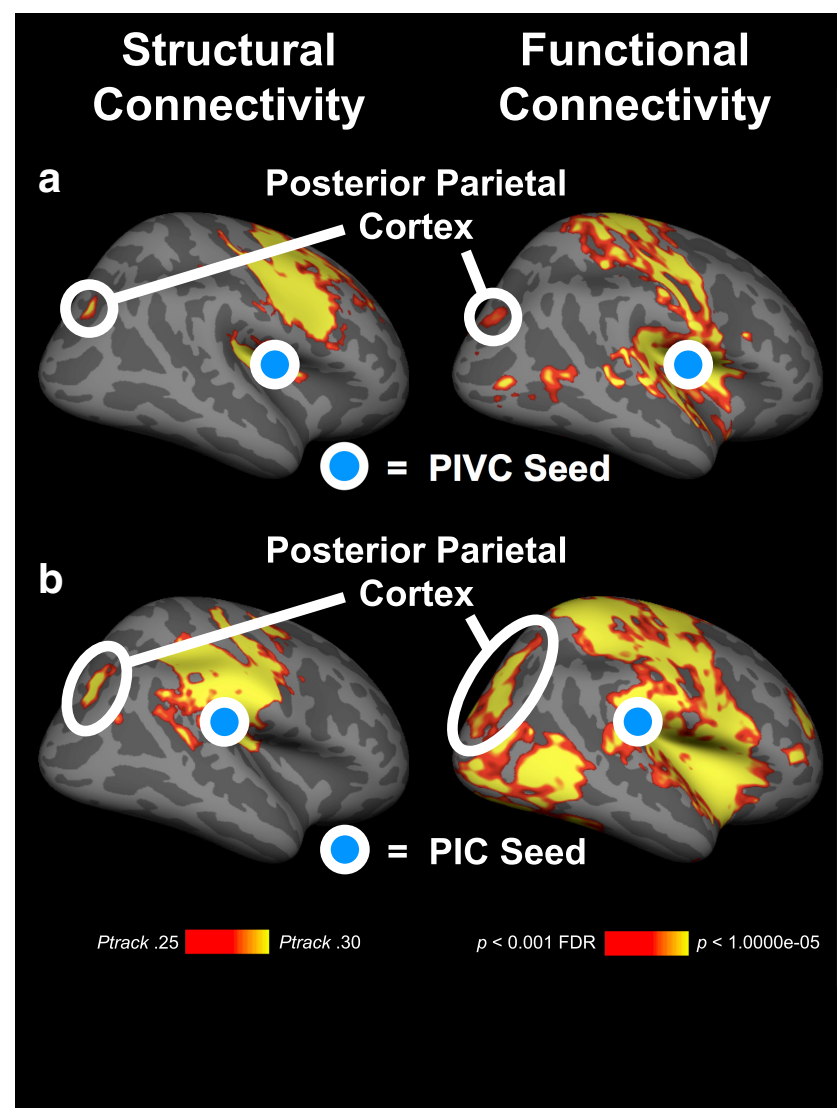

Figure 3. Structural and functional connectivity of PIVC and PIC with PPC. The results are based on group analyses ( $n=20$ subjects) and displayed on the inflated right hemisphere of a template brain. $\boldsymbol{a}$, Connectivity of PIVC (blue disk). Left, Structural connectivity as measured by probabilistic fiber tracking using diffusion weighted imaging. Red-yellow represents the mean probabilities of track terminations from PIVC across subjects. Right, Functional connectivity using resting-state fMRI measurements. Red-yellow represents significant functional connections from PIVC. $\boldsymbol{b}$, Same as in $\boldsymbol{a}$, but for PIC. Structural and functional connections from the PIVC and PIC exist with the PPC.

The resting-state scans were analyzed with a GLM containing the time course of the seed region (PIVC and PIC in the right hemisphere, respectively) as regressor of interest. Additional regressors of no interest in the resting-state GLM were the time courses of the white matter, the ventricles, and the CSF, which were extracted using a principal component analysis, as implemented in the FSFAST processing pipeline.

For each participant, the ROIs were defined on the surface of the inflated right hemisphere of the reconstructed brain at a threshold of $p<$ 0.05 (false discovery rate-corrected) (Frank et al., 2016a). For each subject and hemisphere, PIVC was defined by using activation in the midposterior Sylvian fissure during CVS (see Fig. $4 h$ ). Voxels in PIVC that were also activated in the temperature control experiment (at a threshold of $p<0.05$ false discovery rate-corrected) were removed from the definition of PIVC. The mean number of voxels in the right hemisphere overlapping between CVS and temperature stimulation was 3\% (SE = 9\%). No overlaps between PIC and temperature-related activations were observed in any participant. For a subset of subjects ( $n=10$ subjects of $n=$ 15 subjects in total in the rTMS experiment), we observed that PIC consisted of separate anterior and posterior clusters, similar to previous studies (Frank et al., 2016a, b). However, since this was not the case for each subject, we combined anterior and posterior clusters for all MRI analyses (see Fig. 4i). The mean Talairach coordinates (with SE) for the ROIs (in each case for the right hemisphere) were as follows: PIVC: $x=$ $40( \pm 1), y=-10( \pm 1), z=15$ ( \pm 1$)$; PIC: $x=49( \pm 2), y=-29( \pm 1)$, $z=21( \pm 1)$; parietal rTMS: $x=27( \pm 1), y=-68( \pm 2), z=33( \pm 2)$; occipital rTMS: $x=14( \pm 1), y=-91( \pm 1), z=9( \pm 1)$; sham rTMS: $x=$ $23( \pm 1), y=-88( \pm 1), z=21( \pm 1)$.
DTI analysis. The diffusion data were preprocessed and analyzed as described previously (Wirth et al., 2018). In brief, the FMRIB's Diffusion Toolbox (FDT) (Behrens et al., 2007) was used to correct for head motion and eddy current distortions. Moreover, diffusion vectors were corrected for head motion (Leemans and Jones, 2009). The b-zero images were automatically registered to the reconstructed individual brains. Coregistrations were carefully checked and manually corrected if necessary. For each voxel, a distribution of diffusion parameters was modeled by means of Markov Chain Monte Carlo sampling with two anisotropic compartments unless prevented by automatic relevance detection. Probabilistic tractography was conducted with PIVC and PIC, respectively, in the right hemisphere as the seed for each subject. To this aim, for each vertex in the seed region, 20,000 streamlines (maximum steps $=2000$, step length $=0.5 \mathrm{~mm}$, curvature threshold $=0.2$ ), each based on separate samples of the voxelwise diffusion distribution, were calculated. Tractography was limited to the right hemisphere and tracks were terminated when leaving the hemisphere. Circular pathways were prevented and distance corrections were applied. Track frequencies corresponding to the accumulated number of streamlines were converted to track probabilities $\left(\mathrm{P}_{\text {track }}\right)$ by dividing the log-scaled track frequency by the maximum log-scaled track frequency (Wirth et al., 2018). For the display of cortical track terminations, track probabilities at voxels $1 \mathrm{~mm}$ below the white/gray matter boundary were projected onto the cortical surface for each subject (Beer et al., 2013). Group results were displayed at a threshold of $\mathrm{P}_{\text {track }}=0.25$ commensurate with previous work (Wirth et al., 2018).

Statistical analysis. The sample size of this study was determined based on previous studies (Brandt et al., 1998; Cuturi and MacNeilage, 2014; Frank et al., 2016a,b; Solomon-Harris et al., 2016; Roberts et al., 2017; Schindler and Bartels, 2018; Wirth et al., 2018). Behavioral data (i.e., accuracy on the attentional tracking task and ratings on Likert scales) were analyzed using nonparametric statistics (Friedman's ANOVA, followed by post hoc Wilcoxon-signed rank tests). All other data were analyzed using parametric statistics (repeated-measures ANOVA, followed by post hoc paired-sample $t$ tests). When the assumption of sphericity for the ANOVA was violated (as shown by Mauchly's test of sphericity), the Huynh-Feldt correction was used. For all statistical tests, the two-tailed $\alpha$ level was set to 0.05 . We report the following measures of effect size for different statistical tests: $r$ for Wilcoxon signed-rank tests, partial $\eta^{2}$ for parametric ANOVAs, and Cohen's $d$ for $t$ tests.

\section{Results}

In the first behavioral experiment, subjects received vestibular cues by means of CVS while keeping their eyes closed, passively viewing moving visual stimuli, or attentively tracking a subset of the moving visual stimuli (Fig. 1a). At the end of each trial, subjects rated the magnitude of their vestibular sensations during the trial.

Subjects' vestibular sensations were significantly different between eyes closed, passive viewing, and attentive tracking conditions (Friedman's ANOVA, $\chi_{(2)}^{2}=20.4, p<0.001$ ) (Fig. 1b). Specifically, post hoc Wilcoxon signed-rank tests showed that vestibular sensations were significantly greater in the eyes-closed condition compared with passive viewing $(Z=-3.13, p=0.002, r=$ $-0.57)$ and attentive tracking $(Z=-3.35, p<0.001, r=-0.61)$, suggesting that the processing of visual stimuli both with and without attention decreased vestibular sensations. Importantly, vestibular sensations were significantly weaker during attentive tracking compared with passive viewing $(Z=-3.08, p=0.002$, $r=-0.56$ ), suggesting that the suppression of vestibular sensations was greater in magnitude when attention was directed toward visual processing. Participants achieved a mean accuracy of $91 \%$ correct $(\mathrm{SE}=1 \%$ ) on the attentional tracking task. They sensed self-motion primarily in the yaw direction (on average, in $57 \%$ of all trials, $\mathrm{SE}=2 \%$ ), followed by roll (on average, in $25 \%$ 
of all trials, $\mathrm{SE}=2 \%$ ), whereas pitch or a combination of different directions were reported in the remaining trials.

In the next experiment, we examined activity in core areas of the vestibular cortex (the areas PIVC and PIC, respectively; Fig. $2 a$ ) during passive viewing and attentive tracking of visual motion cues. A $2 \times$ 2 repeated-measures ANOVA with the factors of brain area (PIVC, PIC) and visual condition (passive viewing, attentive tracking) yielded a significant two-way interaction between brain area and visual condition $\left(F_{(1,19)}=71.2, p<0.001\right.$, partial $\left.\eta^{2}=0.79\right)$, indicating that differential activity changes between PIVC and PIC were augmented in the attentive tracking compared with the passive viewing condition (Fig. $2 c, d$ ). Furthermore, there was a significant main effect of brain area $\left(F_{(1,19)}=86.9, p<0.001\right.$, partial $\eta^{2}=$ $0.82)$, indicating that activity changes from baseline were in opposite directions between PIVC and PIC. There was no significant main effect of visual condition $\left(F_{(1,19)}=0.35, p=0.56\right.$, partial $\eta^{2}=$ $0.02)$. Post hoc paired-sample $t$ tests between activity during attentive tracking and passive viewing showed that PIVC was more strongly inhibited during attentive tracking compared with passive viewing $\left(t_{(19)}=-4.52, p<0.001, d=-1.01\right)$. This effect was specific to PIVC and did not occur in core cortical areas of other sensory systems (e.g., the primary somatosensory or auditory cortex; Fig. $2 b$ ). On the contrary, PIC was more strongly activated in attentive tracking compared with passive viewing $\left(t_{(19)}=5.87, p<0.001, d=1.31\right)$. Participants achieved a mean accuracy of $89.2 \%$ correct $(\mathrm{SE}=1.46 \%)$ across runs on the attentional tracking task. The differential activity changes in PIVC and PIC during attentive tracking compared with passive viewing replicate previously reported results by Frank et al. (2016a).

The results of this experiment suggest that attention can modulate the magnitude of activity change in core areas of the vestibular cortex during visual processing. Specifically, compared with passive viewing, attentive visual processing increased the magnitude of inhibition of PIVC and of activity enhancement of the visual-vestibular PIC. Furthermore, the results of this experiment suggest that the attention-induced inhibition is specific to PIVC and does not include core areas of other sensory cortices.

In the next two experiments, we aimed to identify the cortical areas from which the attentional modulation originates. First, the structural and functional connectivity from PIVC and PIC, respectively, was measured. The results showed that PIVC and PIC shared structural and functional connectivity with the PPC (Fig. 3), a central region of the cortical attention network (Culham et al., 2001; Carrasco, 2011). Across subjects, the connected subsection of the parietal cortex was located in the intraparietal sulcus and the surrounding cortex (Brodmann areas 7 and 19) and overlapped with the locations of areas IPS1, V6A, and V7, as defined by the multimodal classification of the cerebral cortex by Glasser et al. (2016) (Fig. 4). These results suggest that the PPC might be the origin of the attentional modulation of activity in core vestibular areas.

We examined this hypothesis in a fourth experiment by using inhibitory rTMS over PPC to reduce its potential modulatory influence over the vestibular cortex, thus reducing the inhibition of PIVC as well as reducing the activity enhancement of PIC during visual attentional tracking. Inhibitory rTMS was applied in three randomized separate sessions over the PPC, the OC, and a control region located in between these two brain areas for sham rTMS (Figs. 4, Fig. 5a).

If rTMS successfully inhibited PPC and OC, behavioral performance on the tracking task should decrease compared with the sham control condition because PPC and OC are both critical to performing attentional tracking (Culham et al., 2001; Frank et al., 2016a). A Friedman's ANOVA across subjects' attentional tracking performance in the three rTMS conditions revealed significant differences between the rTMS conditions $\left(\chi_{(2)}^{2}=6.20, p=0.045\right)$ (Fig. $5 b)$. Post hoc Wilcoxon signed-rank tests showed that performance after parietal rTMS was significantly lower compared with sham rTMS $(Z=-2.51, p=0.01, r=-0.46)$. A similar, marginally significant trend but with moderate effect size was observed for rTMS over OC $(Z=-1.85, p=0.065, r=-0.34)$. Performance did not differ significantly between rTMS over PPC and OC $(Z=-0.67$, $p=0.50, r=-0.12)$. These results show that rTMS decreased subjects' attentional tracking performance, indicating that functional processing in the PPC and OC was impaired after inhibitory rTMS.

A $3 \times 2 \times 2$ repeated-measures ANOVA with the factors of rTMS condition (sham, PPC, OC), visual condition (passive 


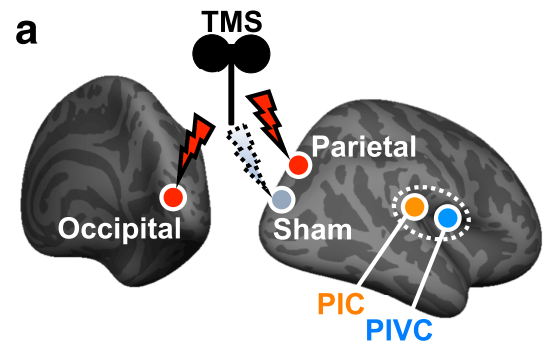

b
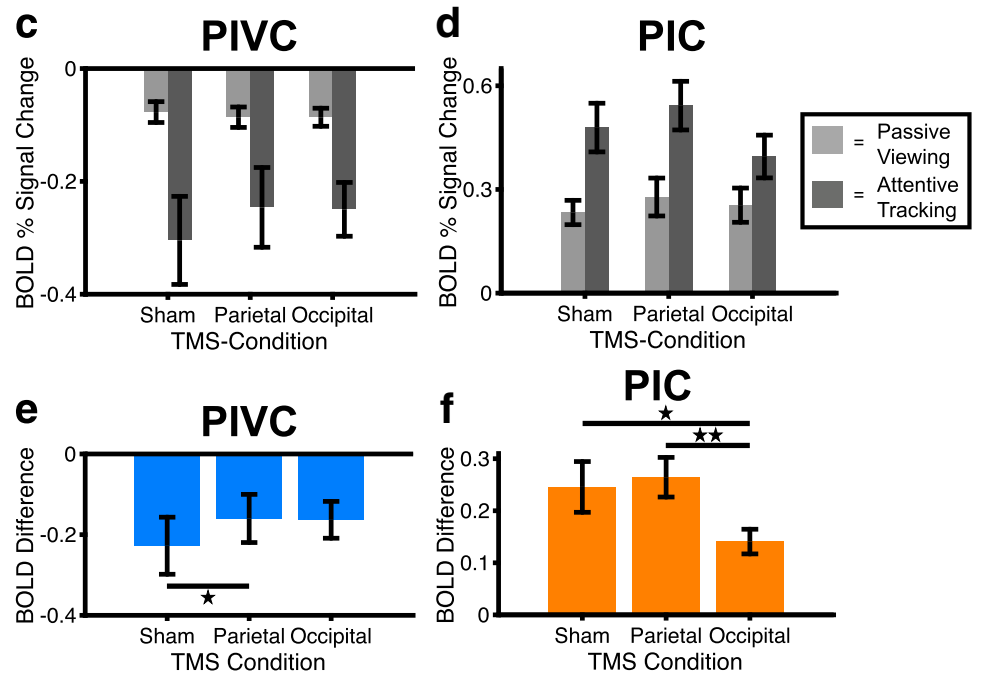

Figure 5. Effects of rTMS on attentional tracking performance and BOLD. $\boldsymbol{a}$, Locations in PPC and OC for inhibitory rTMS. An intermediately located region was chosen for sham rTMS (for the anatomical locations, see Fig. 4). $\boldsymbol{b}$, Mean ( \pm SE) behavioral performance during attentive tracking after inhibitory rTMS ( $n=15$ subjects). $\boldsymbol{c}, \boldsymbol{d}$, Mean ( \pm SE) BOLD activity in PIVC and PIC during passive viewing (light gray) and attentive tracking (dark gray) after inhibitory rTMS ( $n=15$ subjects). Zero on the $y$ axis indicates activity during blank baseline while participants kept their eyes open. $\boldsymbol{e}, \boldsymbol{f}$, Mean ( \pm SE) attention-modulated BOLD activity in PIVC and PIC following inhibitory rTMS ( $n=15$ subjects). Values on the $y$ axis correspond to the activity difference between attentive tracking and passive viewing (see $\boldsymbol{c}, \boldsymbol{d})$. Significant differences between conditions: ${ }^{*} p<0.05,{ }^{* *} p<0.01$.

viewing, attentive tracking), and brain area (PIVC, PIC) was conducted on fMRI activity changes following rTMS (Fig. $5 c, d$ ). Most importantly, there was a significant three-way interaction between rTMS condition, visual condition, and brain area $\left(F_{(2,28)}=5.83, p=0.008\right.$, partial $\left.\eta^{2}=0.29\right)$, suggesting that inhibitory rTMS over PPC and OC reduced the influence of attention on PIVC and PIC, whereas sensory-driven activity changes induced by passive visual processing remained unaffected (Fig. $5 c, d$ ). Furthermore, there was a significant main effect of brain area $\left(F_{(1,14)}=121.7, p<0.001\right.$, partial $\left.\eta^{2}=0.90\right)$ and a significant two-way interaction between visual condition and brain area $\left(F_{(1,14)}=96.1, p<0.001\right.$, partial $\left.\eta^{2}=0.87\right)$, thus replicating the differential activity changes in PIVC and PIC during passive viewing and attentive tracking (see Fig. $2 c, d$ ). No other main effects or interactions were significant. A control analysis for which all attentional tracking trials (i.e., also trials with incorrect subject responses with respect to the tracking task) were included showed a similar three-way interaction between rTMS, visual condition, and brain area $\left(F_{(1.53,21.4)}=6.13, p=\right.$ 0.01 , partial $\eta^{2}=0.30$ ).

Post hoc paired-sample $t$ tests on the activity differences between attentive tracking and passive viewing were conducted to illustrate the effects of inhibitory rTMS on attention-modulated activity changes in the vestibular cortex. The results show that the inhibition of PIVC was significantly weaker after rTMS over PPC compared with sham rTMS $\left(t_{(14)}=2.77, p=0.02, d=0.72\right.$ ) (Fig. $5 e$ ). On the contrary, the inhibition of PIVC after rTMS over OC was not significantly different from sham stimulation $\left(t_{(14)}=\right.$
$1.08, p=0.30, d=0.28)$. The enhancement of activity of PIC was significantly less pronounced after rTMS over OC compared with sham stimulation $\left(t_{(14)}=\right.$ $-2.15, p=0.049, d=-0.56)$ as well compared with rTMS over PPC $\left(t_{(14)}=\right.$ -3.47, $p=0.004, d=-0.89$ ) (Fig. 5f). These results indicate that inhibitory rTMS over PPC reduced the attentionmodulated inhibition of PIVC, whereas rTMS over OC reduced the attentionmodulated activity enhancement of PIC.

\section{Discussion}

Our results support a critical role of attention networks in the parietooccipital cortex for modulating activity of the vestibular cortex when visual processing is prioritized. Although it has been observed that PIVC, a core area of the vestibular cortex, is inhibited during visual processing (Brandt et al., 1998; Deutschländer et al., 2002; Kleinschmidt et al., 2002; Frank et al., 2016a,b), it has remained unclear how the inhibition is modulated in magnitude and from where in the brain this modulatory influence originates (Brandt and Dieterich, 1999; Frank and Greenlee, 2018).

In a previous study, we observed that PIVC became more strongly inhibited the greater the attentional load was on the visual system, suggesting a possible connection between visual attention and crossmodal inhibition of the vestibular system (Frank et al., 2016a). Here, we find support that visual attention can modulate activity in core areas of the vestibular cortex and that this modulatory influence originates from attention networks in the parietooccipital cortex. Specifically, we find that vestibular sensations of self-motion were suppressed to a greater extent when visual stimuli were processed attentively rather than passively, suggesting that attention increased the magnitude of vestibular suppression. This interpretation is supported by our findings with fMRI showing that the inhibition of PIVC increased when visual stimuli were processed attentively rather than passively. The cortical origin of this modulatory influence of visual attention over PIVC appeared to be the PPC, which we found to be structurally and functionally connected with PIVC. Specifically, inhibitory rTMS over PPC reduced the magnitude of inhibition of PIVC during attentive visual processing, suggesting that PPC exerted less modulatory influence over PIVC. For PIC, a visual-vestibular area located posterior to PIVC, we found that activity increased when visual stimuli were processed attentively and that this enhancement of activity was associated with activity in attention networks in the OC.

Previous studies found that the core of the vestibular cortex is inhibited during visual processing (Brandt et al., 1998; Deutschländer et al., 2002; Kleinschmidt et al., 2002). However, these studies did not differentiate between different subregions within the core of the vestibular cortex. Here, we have replicated the inhibition of PIVC but additionally found that PIC, a visualvestibular area located in close vicinity posterior to PIVC (Frank 
et al., 2014, 2016b; Frank and Greenlee, 2018), exhibited enhanced activity when subjects attentively tracked visual motion cues, corroborating previous findings (Frank et al., 2016a). Our rTMS results suggest that the enhancement of activity in PIC is associated with activity in the OC. The OC is subject to strong influence by attention, potentially originating from higher-order attention networks (Carrasco, 2011). Since PIC is strongly associated with the visual network (Frank et al., 2016b), we speculate that this attention-modulated enhancement of activity in the OC during attentive visual tracking is propagated to PIC.

Our results are limited in that they are based on the assumption of direct connectivity between PPC and the core of the vestibular cortex. Although there is evidence supporting the existence of such a pathway (Uesaki et al., 2018; Wirth et al., 2018; Dionisio et al., 2019; for nonhuman primates: Guldin and Grüsser, 1998; for review, see Lopez and Blanke, 2011) (see Fig. 3 ), the attentional modulation of activity in the vestibular cortex might also emerge through corticofugal projections from PPC to the thalamus or the vestibular nuclei in the brainstem (FaugierGrimaud and Ventre, 1989). If the thalamus or the vestibular nuclei are inhibited by PPC, fewer excitatory signals will reach the PIVC; and, as a result, the activity of the PIVC will be reduced. Future studies are necessary to disambiguate the possibilities of corticocortical or corticofugal projections as the underlying neuroanatomical substrate of attentional modulation of the vestibular cortex.

Our study is further limited in that we only investigated changes of activity of the vestibular cortex when visual processing was prioritized, begging the question of whether attention can exert its modulatory influence also in the opposite direction, that is, modulating activity in the visual system when vestibular processing is prioritized. Although there is evidence for the inhibition of the visual cortex during stimulation of the vestibular cortex (Wenzel et al., 1996; Deutschländer et al., 2002; Seemungal et al., 2013; Mazzola et al., 2014), the cross-modal effects of attention directed toward vestibular processing on the visual cortex have not yet been measured. Finally, future studies are necessary to investigate whether attention also modulates the inhibition between other sensory systems, for instance, between the visual and auditory systems (Laurienti et al., 2002) and between the visual and somatosensory systems (Merabet et al., 2007).

In conclusion, our results suggest that attention can play a critical role in modulating activity of the vestibular system when visual processing is prioritized. Furthermore, our results indicate that this modulatory influence emerges from attention networks in the parietooccipital cortex. By modulating activity in the vestibular system, attention might shield the ongoing processing of the prevailing visual cues from potentially conflicting vestibular signals.

\section{References}

Barnes G (1995) Adaptation in the oculomotor response to caloric irrigation and the merits of bithermal stimulation. Br J Audiol 29:95-106.

Beer AL, Plank T, Meyer G, Greenlee MW (2013) Combined diffusionweighted and functional magnetic resonance imaging reveals a temporaloccipital network involved in auditory-visual object processing. Front Integr Neurosci 7:5.

Behrens TE, Berg HJ, Jbabdi S, Rushworth MF, Woolrich MW (2007) Probabilistic diffusion tractography with multiple fibre orientations: what can we gain? Neuroimage 34:144-155.

Berger DR, Bülthoff HH (2009) The role of attention on the integration of visual and inertial cues. Exp Brain Res 198:287-300.

Billington J, Smith AT (2015) Neural mechanisms for discounting headroll-induced retinal motion. J Neurosci 35:4851-4856.

Brainard DH (1997) The psychophysics toolbox. Spat Vis 10:433-436.
Brandt T, Dieterich M (1999) The vestibular cortex: its locations, functions, and disorders. Ann N Y Acad Sci 871:293-312.

Brandt T, Bartenstein P, Janek A, Dieterich M (1998) Reciprocal inhibitory visual-vestibular interaction: visual motion stimulation deactivates the parieto-insular vestibular cortex. Brain 121:1749-1758.

Carrasco M (2011) Visual attention: the past 25 years. Vision Res 51:1484-1525.

Craig AD, Chen K, Bandy D, Reiman EM (2000) Thermosensory activation of insular cortex. Nat Neurosci 3:184-190.

Culham JC, Cavanagh P, Kanwisher NG (2001) Attention response functions: characterizing brain areas using $\mathrm{fMRI}$ activation during parametric variations of attentional load. Neuron 32:737-745.

Cuturi LF, MacNeilage PR (2014) Optic flow induces nonvisual self-motion aftereffects. Curr Biol 24:2817-2821.

Dale AM, Fischl B, Sereno MI (1999) Cortical surface-based analysis: I. Segmentation and surface reconstruction. Neuroimage 9:179-194.

Deutschländer A, Bense S, Stephan T, Schwaiger M, Brandt T, Dieterich M (2002) Sensory system interactions during simultaneous vestibular and visual stimulation in PET. Hum Brain Mapp 16:92-103.

Dieterich M, Bense S, Lutz S, Drzezga A, Stephan T, Bartenstein P, Brandt T (2003) Dominance for vestibular cortical function in the non-dominant hemisphere. Cereb Cortex 13:994-1007.

Dionisio S, Mayoglou L, Cho SM, Prime D, Flanigan PM, Lega B, Mosher J, Leahy R, Gonzalez-Martinez J, Nair D (2019) Connectivity of the human insula: a cortico-cortical evoked potential (CCEP) study. Cortex 120:419-442.

Engel S, Markewitz RD, Langguth B, Schecklmann M (2017) Paired associative stimulation of the temporal cortex: effects on the auditory steadystate response. Front Psychiatry 8:227.

Faugier-Grimaud S, Ventre J (1989) Anatomic connections of inferior parietal cortex (area 7) with subcortical structures related to vestibuloocular function in a monkey (Macaca fascicularis). J Comp Neurol 280:114.

Fischl B, Sereno MI, Dale AM (1999) Cortical surface-based analysis: II: Inflation, flattening, and a surface-based coordinate system. Neuroimage 9:195-207.

Frank SM, Greenlee MW (2014) An MRI-compatible caloric stimulation device for the investigation of human vestibular cortex. J Neurosci Methods 235:208-218.

Frank SM, Greenlee MW (2018) The parieto-insular vestibular cortex in humans: more than a single area? J Neurophysiol 120:1438-1450.

Frank SM, Baumann O, Mattingley JB, Greenlee MW (2014) Vestibular and visual responses in human posterior insular cortex. J Neurophysiol 112: 2481-2491.

Frank SM, Sun L, Forster L, Tse PU, Greenlee MW (2016a) Cross-modal attention effects in the vestibular cortex during attentive tracking of moving objects. J Neurosci 36:12720-12728.

Frank SM, Wirth AM, Greenlee MW (2016b) Visual-vestibular processing in the human sylvian fissure. J Neurophysiol 116:263-271.

Glasser MF, Coalson TS, Robinson EC, Hacker CD, Harwell J, Yacoub E, Ugurbil K, Andersson J, Beckmann CF, Jenkinson M, Smith SM, Van Essen DC (2016) A multi-modal parcellation of human cerebral cortex. Nature 536:171-178.

Guldin WO, Grüsser OJ (1998) Is there a vestibular cortex? Trends Neurosci 21:254-259.

Kleinschmidt A, Thilo KV, Büchel C, Gresty MA, Bronstein AM, Frackowiak RS (2002) Neural correlates of visual-motion perception as object-or self-motion. Neuroimage 16:873-882.

Kobayashi M, Pascual-Leone A (2003) Transcranial magnetic stimulation in neurology. Lancet Neurol 2:145-156.

Laurienti PJ, Burdette JH, Wallace MT, Yen YF, Field AS, Stein BE (2002) Deactivation of sensory-specific cortex by cross-modal stimuli. J Cogn Neurosci 14:420-429.

Leemans A, Jones DK (2009) The B-matrix must be rotated when correcting for subject motion in DTI data. Magn Reson Med 61:1336-1349.

Lopez C, Blanke O (2011) The thalamocortical vestibular system in animals and humans. Brain Res Rev 67:119-146.

Marx E, Deutschländer A, Stephan T, Dieterich M, Wiesmann M, Brandt T (2004) Eyes open and eyes closed as rest conditions: impact on brain activation patterns. Neuroimage 21:1818-1824.

Mazzola L, Lopez C, Faillenot I, Chouchou F, Mauguière F, Isnard J (2014) 
Vestibular responses to direct stimulation of the human insular cortex. Ann Neurol 76:609-619.

Merabet LB, Swisher JD, McMains SA, Halko MA, Amedi A, Pascual-Leone A, Somers DC (2007) Combined activation and deactivation of visual cortex during tactile sensory processing. J Neurophysiol 97:1633-1641.

Oldfield RC (1971) The assessment and analysis of handedness: the Edinburgh Inventory. Neuropsychologia 9:97-113.

Pelli DG (1997) The VideoToolbox software for visual psychophysics: transforming numbers into movies. Spat Vis 10:437-442.

Pylyshyn ZW, Storm RW (1988) Tracking multiple independent targets: evidence for a parallel tracking mechanism. Spat Vis 3:179-197.

Rafique SA, Solomon-Harris LM, Steeves JK (2015) TMS to object cortex affects both object and scene remote networks while TMS to scene cortex only affects scene networks. Neuropsychologia 79:86-96.

Roberts RE, Ahmad H, Arshad Q, Patel M, Dima D, Leech R, Seemungal BM, Sharp DJ, Bronstein AM (2017) Functional neuroimaging of visuovestibular interaction. Brain Struct Funct 222:2329-2343.

Rossi S, Hallett M, Rossini PM, Pascual-Leone A (2009) Safety, ethical considerations, and application guidelines for the use of transcranial magnetic stimulation in clinical practice and research. Clin Neurophysiol 120:2008-2039.

Rossini PM, Burke D, Chen R, Cohen LG, Daskalakis Z, Di Iorio R, Di Lazzaro V, Ferreri F, Fitzgerald PB, George MS, Hallett M, Lefaucheur JP, Langguth B, Matsumoto H, Miniussi C, Nitsche MA, Pascual-Leone A, Paulus W, Rossi S, Rothwell JC, et al. (2015) Non-invasive electrical and magnetic stimulation of the brain, spinal cord, roots and peripheral nerves: basic principles and procedures for routine clinical and research application. an updated report from an IFCN committee. Clin Neurophysiol 126:1071-1107.

Ruff CC, Driver J, Bestmann S (2009) Combining TMS and fMRI: from 'virtual lesions' to functional network accounts of cognition. Cortex 45:1043-1049.

Schecklmann M, Landgrebe M, Frank E, Sand PG, Eichhammer P, Hajak G, Langguth B (2012) Is motor cortex excitability associated with personality factors? A replication study. Int J Psychophysiol 83:323-327.

Schindler A, Bartels A (2018) Integration of visual and non-visual selfmotion cues during voluntary head movements in the human brain. Neuroimage 172:597-607.

Seemungal BM, Guzman-Lopez J, Arshad Q, Schultz SR, Walsh V, Yousif N (2013) Vestibular activation differentially modulates human early visual cortex and V5/MT excitability and response entropy. Cereb Cortex 23:12-19.

Solomon-Harris LM, Rafique SA, Steeves JK (2016) Consecutive TMSfMRI reveals remote effects of neural noise to the "occipital face area." Brain Res 1650:134-141.

Thut G, Pascual-Leone A (2010) A review of combined TMS-EEG studies to characterize lasting effects of repetitive TMS and assess their usefulness in cognitive and clinical neuroscience. Brain Topogr 22:219-232.

Uesaki M, Takemura H, Ashida H (2018) Computational neuroanatomy of human stratum proprium of interparietal sulcus. Brain Struct Funct 223:489-507.

Van Doren J, Langguth B, Schecklmann M (2015) TMS-related potentials and artifacts in combined TMS-EEG measurements: comparison of three different TMS devices. Clin Neurophysiol 45:159-166.

Wenzel R, Bartenstein P, Dieterich M, Danek A, Weindl A, Minoshima S, Ziegler S, Schwaiger M, Brandt T (1996) Deactivation of human visual cortex during involuntary ocular oscillations: a PET activation study. Brain 119:101-110.

Wirth AM, Frank SM, Greenlee MW, Beer AL (2018) White matter connectivity of the visual-vestibular cortex examined by diffusion-weighted imaging. Brain Connect 8:235-244. 